\title{
Personalia
}

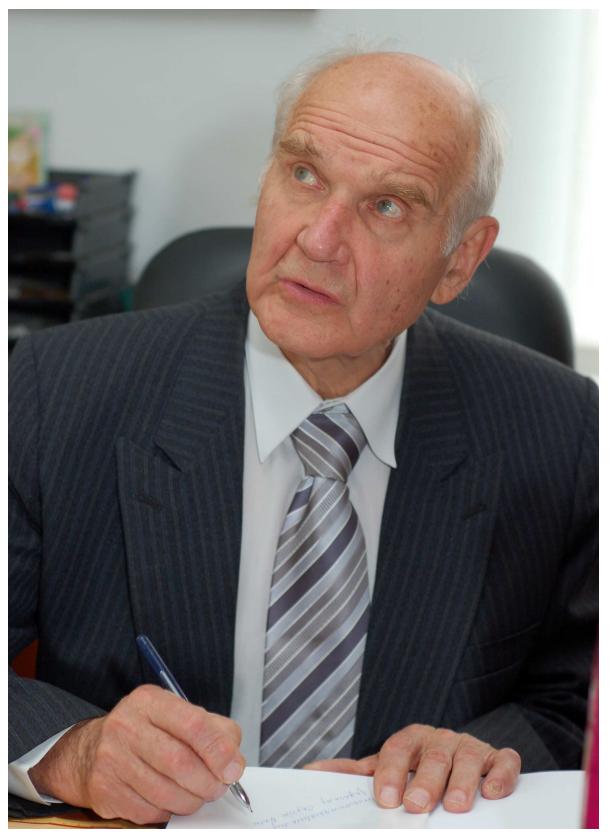

\section{Ihor Yukhnovskii's 90th birthday}

On September 1, 2015 a prominent Ukrainian scholar, theoretical physicist, politician and a public figure, full member of the National Academy of Sciences, Doctor of Sciences in mathematics and physics, Professor, and permanent Editor-in-Chief of the journal "Condensed Matter Physics" Ihor Yukhnovskii celebrates his 90th birthday. During his eventful life he created a lot: from methods and new approaches in statistical physics and condensed matter theory to scientific and research structures including the Institute for Condensed Matter Physics of the National Academy of Sciences and the journal "Condensed Matter Physics”. He meets his birthday anniversary being full of energy, with new ideas and plans.

Ihor Yukhnovskii was born in Knyahynin, Rivne region, to a family of state employees which, in turn, were descendants from the Priest families. He graduated from Lyceum in Kremenets, Ternopil region and was recruited in 1944 and then went through the warfare routes of Ukraine, Poland and Austria. After the World War II (in 1946), Ihor Yukhnovskii entered the Ivan Franko State University of Lviv, the Department of Physics and Mathematics. He graduated from the University in 1951 with Honor Diploma and continued his study as a postgraduate student at the Chair of Theoretical Physics. In early post-war years, famous books were published by academician N.N. Bogolyubov, namely "Problems of Dynamic Theory in Statistical Physics" (1946) and "Lectures on Quantum Statistics” (1949) which, in fact, defined the trend of scientific interests of I.R. Yukhnovskii. Having studied these books, he decided to become a theoretical physicist and to be engaged in statistical physics. Ihor Yukhnovskii followed the Bogolyubov line in all his future studies and, on this basis, founded the Lviv school of Statistical Physics.

The first scientific papers by I.R. Yukhnovskii were focused on the development of the Bogolyubov method of plasma parameter expansion for the study of binary distribution functions in charged particle systems. He obtained general formula expressions which contained the screened potentials of Coulomb interactions and, thus, allowed one to correctly take into account the effects of long-range interactions. These results formed the basis for his Ph.D. thesis and turned to be far ahead of the similar research results obtained later, which are now known as a $\gamma$-ordering technique. 
In the late 50ies, I.R. Yukhnovskii formulated a new and powerful method for the study of manyparticle systems, namely, the method of collective variables which later on became the basic method in his research. He proposed an original calculation scheme of the transition function from the Cartesian coordinates of particles to the variables describing collective movements in the system. It made it possible to obtain a functional representation of the partition function in the phase space of collective variables and to take into account the correlations among the fluctuation waves. On this basis, I.R. Yukhnovskii solved another important problem, namely, a simultaneous consideration of both short-range and long-range interactions. In the approach proposed by him, the description of the system of interacting particles is carried out in an extended phase space that includes individual coordinates of particles (a reference system) and collective variables describing the density fluctuation waves. Individual and collective variables describe the effects caused by short- and long-range interactions, respectively. In the mid-60s, he generalized the formalism of collective variables to the case of quantum many-particle systems. An equivalent representation was found for the partition function of a quantum system corresponding formally to the classical many-particle interacting system. This approach, referred to as the method of displacements and collective variables, appeared to be successful for the study of quantum Fermi and Bose systems.

The original results dealing with the systems of charged particles formed the basis for I.R. Yukhnovskii's D.Sc. (Habilitation) thesis "Statistical Theory of the Charged Particle Systems" that was defended in 1965 at the Taras Shevchenko State University of Kyiv.

In 1958-1969 I.R. Yukhnovskii headed the Chair of Theoretical Physics at the Ivan Franko State University of Lviv. In 1969, he set up in Lviv the Department of Statistical Theory of Condensed States (STCS) of the Institute for Theoretical Physics of the Academy of Sciences of Ukraine. In that time, the Institute was led by N.N. Bogolyubov. In 1972 Ihor Yukhnovskii was elected the corresponding member of the Academy of Sciences of Ukraine. In 1980, based on the STCS, the Lviv Division of Statistical Physics of the Institute for Theoretical Physics of the Academy of Sciences of Ukraine was organized. In 1982 I.R. Yukhnovskii became the full member of the Academy of Sciences on the specialization "theoretical physics".

At the same time, the following lines of investigations were developed: the theory of electrolyte solutions, physics of quantum many-particle systems, the theory of phase transitions and critical phenomena.

It was shown within the framework of the theory of electrolyte that the character of the screening of electrostatic interactions caused by ions and molecules of the solvent is fundamentally different: an ionic screening leads to the exponential decay of electrostatic interactions while screening by polar molecules determines the dielectric properties of the solvent. A key role of the molecular subsystem of a solvent, microscopic mechanisms of both ionic solvation and the short-range ordering in solutions were established and studied. The theory of electrolyte solution was then extended to the instance of spatially bounded systems (electrolyte films and membranes). It was shown that screening effects in spatially nonuniform and bulk systems are qualitatively different due to the presence of electrostatic reflection forces in semibounded systems. These studies were carried out in collaboration with M.F. Holovko, V.S. Vysochanskii, I.Y. Kuryliak, O.O. Pizio, Ye.M. Soviak, A.F. Kovalenko et al.

The formulation of the method of displacements and collective variables caused active investigations of quantum systems of interacting particles. The main results obtained in this direction are as follows: equation of state of a degenerate electron gas, binary distribution functions of electrons in a regime of strong nonideality, correct asymptotic behaviour of the binary distribution function at small distances for electron density typical of metals (with M.V. Vavrukh, G.I. Bigun, P.M. Petrashko, P.P. Kostrobij). The study of high-temperature plasma was conducted together with L.F. Blazhiyevskii. I.R. Yukhnovskii, I.O. Vakarchuk and O.L. Gonopolskii developed the microscopic theory of liquid $\mathrm{He}^{4}$ that produced quantitative agreement of the theoretical results with experimental data. The idea of a reference system proposed by I.R. Yukhnovskii was extended to the quantum systems of the order-disorder type described by pseudo-spin models (with R.R. Levitskii).

The development of a microscopic theory of phase transitions has a special place in Yukhnovskii's scientific life. Originated in early 1970-ies, his interest in this subject has not weakened today. The first papers by I.R. Yukhnovskii in this line were published in the 1970-ies and already contained the basic ideas of the original method for calculating a partition function of the three-dimensional Ising model near the second order phase transition. In these and subsequent papers it was shown that the non-Gaussian distributions of fluctuations of the order parameter should be used for a correct description of critical phenomena. It was also established that a special critical regime is valid in the vicinity of the critical point, where 
a new type of symmetry, i.e., the renormalization group symmetry, emerges. This leads to a universal behaviour of different systems. These investigations were carried out in collaboration with Yu.K. Rudavskii, M.P. Kozlovskii, I.O. Vakarchuk, V.O. Kolomiets, Yu.V. Holovatch, I.M. Mryglod. At the same time, it was shown that by taking into account the critical regime alone, one cannot satisfactorily describe the correct behaviour of thermodynamic properties of the system near the phase transition point. Together with M.P. Kozlovskii, I.R. Yukhnovskii proposed the method for the calculation of universal and non-universal quantities of the Ising model. This approach provided a key for the construction of the general theory of critical phenomena in three dimensional systems and then applied by Yukhnovskii's disciples to the study of the critical behaviour in various models of statistical physics and theory of condensed matter: a $n$-component spin model (with I.O. Vakarchuk, Yu.K. Rudavskii, Yu.V. Holovatch), binary substitution alloys (with Z.O. Gurskii), a $n$-component model of structural phase transition (with I.M. Mryglod), Ising systems with anisotropic interactions (with M.A. Korynevskii), a gas-liquid critical point (with I.M. Idzyk and V.O. Kolomiyets), classical multicomponent mixtures (with O.V. Patsahan).

The extension of ideas of the collective variable method to the problems of non-equilibrium statistical physics enabled I.R. Yukhnovskii's disciples to obtain completely new results in the dynamics of dense fluids and liquid systems (M.V. Tokarchuk, I.M. Mryglod, T.M. Bryk, I.P. Omelyan).

All these achievements enabled I.R. Yukhnovskii to set up in 1990, on the basis of the Lviv Devision of Statistical Physics of the Institute for Theoretical Physics, the Institute for Condensed Matter Physics of the National Academy of Sciences of Ukraine which he headed for 16 years. The efforts made by Yukhnovskii I.R. and by his numerous followers resulted in the formation of the Lviv school of Statistical Physics known today throughout the world. Within the Lviv school there have been developed several original and powerful methods for theoretical investigations of the systems of interacting particles which enables one to solve many important problems of Condensed Matter Physics. Members of the school made a substantial contribution to the theory of liquids and electrolyte solutions, metals and alloys, disordered systems, phase transitions and critical phenomena. Among the scientific interests of I.R. Yukhnovskii there also were the mathematical methods in economics and society evolution, the security problems of "Shelter" site in the Chornobyl nuclear power plant. I.R. Yukhnovskii is the author of over 500 research papers, 7 monographs and textbooks.

In 2010, after over 20 years of political and state activities in Kyiv, I.R. Yukhnovskii came back to Lviv and returned to science. Today the academician I.R. Yukhnovskii is an advisor of the Presidium of National Academy of Sciences of Ukraine, a honorary director of the Institute for Condensed Matter Physics. Very recently, at the Jubilee Lectures "Statistical Physics in the 21st Century" (Tatariv, 26-28 of August, 2015) he presented his new results on the investigation of the gas-liquid phase transition near the critical point. The results attracted interest, stimulated discussions and raised new questions. During these Lectures I.R. Yukhnovskii together with colleagues-physicists climbed Hoverla, the highest mountain of Ukraine. The Editorial Board of "Condensed Matter Physics" and collaborators of the Institute for Condensed Matter Physics congratulate I.R. Yukhnovskii on the occasion of his anniversary and wish him to stay in good health and conquer many new peaks. 
